\title{
Autosomal recessive cerebellar ataxia due to STUB1 deficiency
}

INSERM

\section{Source}

INSERM. (1999). Orphanet: an online rare disease and orphan drug data base. Autosomal recessive cerebellar ataxia due to ST UB1 deficiency. ORPHA:412057

Autosomal recessive cerebellar ataxia due to ST UB1 deficiency is a rare hereditary ataxia characterized by progressive truncal and limb ataxia resulting in gait instability.

Dysarthria, dysphagia, nystagmus, spasticity of the lower limbs, mild peripheral sensory neuropathy, cognitive impairment and accelerated ageing have also been associated. 